\title{
Ten Years at the Helm of Communications of the ACM
}

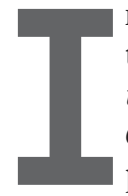

N JANUARY 2007, I undertook the task of revitalizing Communications of the ACM. In the 1970s, Communications was one of the premier publications in computing, publishing seminal articles such as Codd's "A Relational Model of Data for Large Shared Data Banks" (June 1970) and Rivest, Shamir, and Adleman's “A Method for Obtaining Digital Signature and Public-Key Cryptosystems" (February 1978). By the 1990s, however, Communications' content had evolved to be strongly slanted toward Management Information Systems. Over time, a significant segment of the ACM membership had lost interest in the publication. When David Patterson became ACM President in 2005 , he argued that this state of affairs was unacceptable. The revitalized Communications was launched in July 2008, and by March 2009 I was able to conclude "Yes, it can be done" (see https://goo.gl/0ySCMj). Now, after 10 years at the helm of Communications, it is time for me to move on and for the magazine to get new leadership. Expect a formal announcement on this in the very near future!

My 10 years with Communications has been an incredible learning experience. I would like to share one of the most important lessons I learned. To turn Communications around, it was important to understand first what went wrong. How has Communications evolved from a premier publication in computing to one that was of interest only to a narrow segment of the computing community? The answer, in one word, is emergence, the phenomenon whereby a systemwide behavior arises through numerous interactions among system components. An example of emergence is gentrification, which is a process of renovation of deteriorated urban neighborhoods by means of the influx of more affluent residents. The reverse trend is usually called urban decline. Gentrification and urban decline are the result of numerous individual decisions of residents moving into and moving out of the neighborhood. With no central direction, a neighborhood can completely change its character over a couple of decades.

The standard scholarly editorial model is that of filtration: authors submit articles, and editors filter them, with the help of reviewers, based on scope and quality. While the editorial process plays a critical role in shaping the face of a publication, the dominant factor is the nature of the submitted articles, which is determined solely by the submitting authors. Authors make submission decisions to a large measure based on articles already published. Thus, just as a neighborhood can change its character over a couple of decades, a publication can see its character change in just a few years. This is how Communications changed in the 1990s from a magazine representing all of computing to essentially "MIS Monthly."

The key to the turnaround of $\mathrm{Com}^{-}$ munications was to change the editorial model from one mostly based on filtration to one mostly based on curation. The word "curation" comes from the verb "to care" It is typically used in the context of museums, where a curator selects items for exhibits. It also refers more broadly to the process of gathering and selecting content. While Communications is open to submissions, the lion's share of its published content is curated. Certain sections, such as News, Viewpoint columns, and Practice, are purely curated, with an editorial-board section in charge of the curation. Other sections, combine curation and filtration. Take Contributed Articles, for example. During 2016, we received 202 submissions. Most of these articles were unsolicited, but a significant fraction of the submitted articles were solicited, which means that an editor encouraged a specific author to submit an article on a specific topic. Both solicited and unsolicited articles are subject to the same rigorous peer review, but the selection of topics and authors of so- $^{-}$ licited articles results in a higher probability of a positive editorial outcome.

Curation ensures Communications continues to be a publication that is broadly representative of computing. But curation requires an editorial board that is not merely reactive, but is strongly proactive, continually seeking topics and authors for high-quality articles. Communications' Editorial Board consists of approximately 100 committed volunteers. The quality and commitment of the Editorial Board is the real key to the success of the revitalized Communications. For all of Communications' success these past years, it will continue to remain a work in progress that will need the dedicated involvement of members of the community to keep it current and relevant for the fast-changing field of computing. Both ACM and the community will need to continue investing in Communications to keep it strong and vital.

Follow me on Facebook, Google+, and Twitter.

Moshe Y. Vardi, EDITOR-IN-CHIEF

Copyright held by author. 